\title{
REGISTRATION OF DENTAL ATLAS TO RADIOGRAPHS FOR HUMAN IDENTIFICATION
}

\author{
Anil K. Jain, and Hong Chen \\ Department of Computer Science and Engineering \\ Michigan State University \\ East Lansing, MI 48824
}

\begin{abstract}
The human dental atlas contains a detailed description of each tooth in the mouth and their relative positions. Registering a dental radiograph to the dental atlas reveals the position and index of each tooth in the radiograph. This helps in establishing the correspondence of teeth when matching two radiographs for human identification. We propose a hidden Markov model (HMM) as an underlying representation of the dental atlas. In our model, the states representing the available teeth have discrete observations, namely the class of each tooth, and the states representing the missing teeth have continuous observations - the distance between neighboring teeth. To classify the teeth, three support vector machines (SVMs) using different feature sets are combined using the average fusion method. Experimental results show that this registration algorithm is promising.
\end{abstract}

\section{INTRODUCTION}

Dental Biometrics is used to identify deceased people using dental radiographs. In many situations (e.g., fire victims), the conventional biometric features, i.e., face, fingerprint, and iris, may not be available. To conduct human identification, a radiograph taken before the death (Antemortem (AM) radiograph) and a radiograph taken after the death (Postmortem (PM) radiograph) are required. The PM radiograph is matched with the AM radiographs stored in the database. Since the identities of the AM radiographs are already known, if the teeth in the PM radiograph match the teeth in a specific AM radiograph with sufficiently high precision, the identity of the PM radiograph can be established. However, this matching requires that the indices of the teeth in the radiographs are known.

A fully developed adult dental structure contains a total of 32 teeth. Each of the 32 teeth has a specific position and index in the dental atlas. We propose a method to register the dental radiograph images to the dental atlas, so

This research was supported by the National Science Foundation grant EIA-0131079. that the index of each tooth in the radiographs can be estimated. This will help in matching AM and PM radiographs. Mahoor and Abdel-Mottaleb [1] proposed a method to obtain the indices of teeth using bitewing images. A major limitation of their method is that bitewing images contain only two classes of teeth: molars and bicuspids. Further, they assume that there are no missing teeth in the images. When this assumption is not satisfied or when there are classification errors, their string matching algorithm will result in registration errors. Our proposed method deals with all three types of dental radiographs: bitewing, periapical, and panoramic images (Fig. 1), and the teeth are classified into three classes: molars, (bi)cuspids, and incisors (Fig. 2) [2]. Our method uses a hidden Markov model (HMM) to register the teeth sequence, so that the missing teeth can be detected, and the classification errors can then be reduced using the model.

\section{A MODEL OF DENTAL ATLAS}

The dental atlas of a full set of adult teeth is shown in Fig. 3. In Fig. 4, the hidden Markov model (HMM) representing only the 16 upper teeth is shown. Since some teeth may be missing, to model a single position in the dental atlas, a state (tooth state) representing an observered tooth is combined with three states (distance state) that represent 0,1 , or 2 missing teeth. The tooth states are shown as circles, and the numbers inside the circles are the indices of the teeth they represent. The distance states are shown as squares, and the numbers inside the squares are the numbers of missing teeth. Here we assume there are at most 2 missing teeth between any pair of neighboring teeth in a sequence. The HMM representing the lower row of teeth has the same structure as the model shown in Fig. 4, except that the tooth indices inside tooth states now go from 32 down to 16 .

A HMM requires one observation for each state. The observation for a tooth state can be either a molar, a (bi)cuspid, or an incisor. The method for classification of teeth using their contours is presented in section III. The observations 


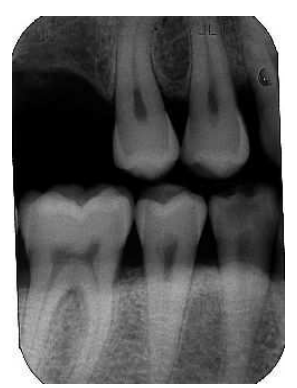

(a)

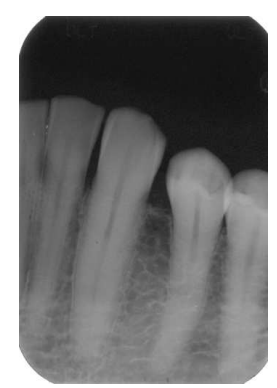

(b)

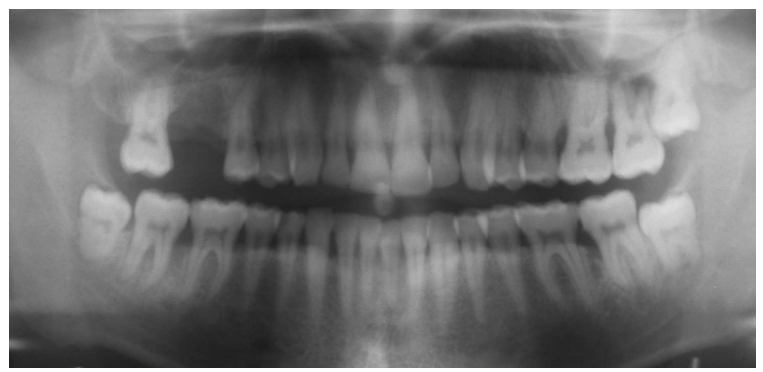

(c)

Fig. 1. Three types of dental radiograph images. (a) Bitewing image; (b) periapical image; (c) panoramic image.

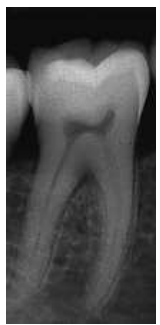

(a)

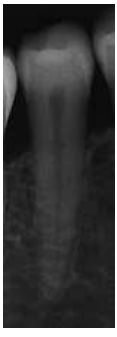

(b)

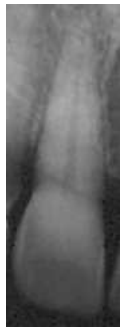

(c)
Fig. 2. Three types of tooth. (a) Molar; (b) (bi)cuspid; (c) incisor.

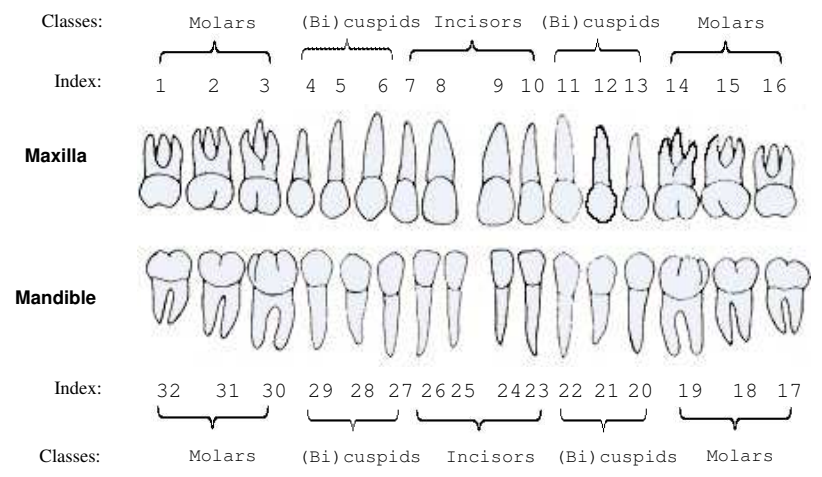

Fig. 3. Dental Atlas of a full set of adult teeth containing the indices and classification labels of the teeth.
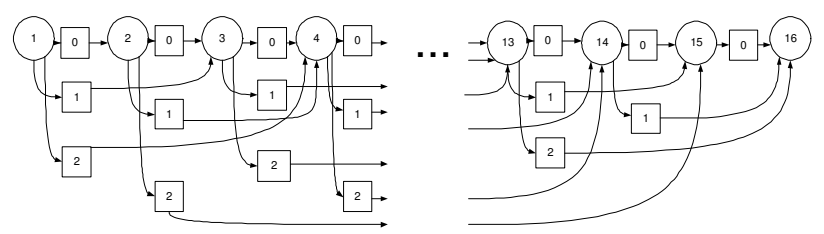

Fig. 4. Hidden Markov model for upper row of teeth.

for a distance state is the distance between the neighboring teeth. So, the observation sequences are in the form of \{tooth type, distance, tooth type, distance, tooth type, ..., tooth type $\}$. Since the radiographs have different resolutions, to normalize the inter-tooth distances to the same scale, each distance is divided by the width of the left tooth in the two neighboring teeth. Fig. 5 shows the procedure for generating an observation sequence. Let the observed sequence be denoted as $B=\left\{a_{1}, b_{1}, a_{2}, b_{2}, \ldots, a_{n}\right\}$, where $a_{i}, i=1, \ldots, n$, is the type of the $i$ th tooth in the radiograph, and $b_{i}, i=1, \ldots, n-1$, is the distance between the $i$ th tooth and the $(i+1)$ th tooth. If the HMM model is denoted as $Q$, the goal of registration is to find the path $K=\left[k_{1}, k_{2}, k_{3}, \ldots, k_{2 n-1}\right]$, where $k_{i}$ is a tooth state when $i$ is even, and a distance state when $i$ is odd. $K$ satisfies that

$$
P(B, K)=\max _{\text {All possible } K \text { in } Q} P(B \mid K) P(K),
$$

where $P(B \mid K)$ is the probability of generating the observation sequence $B$ given path $K$, and $P(K)$ is the probability of forming path $K$. Due to the first-order Markov assumption [3], the probability of transition to current state depends only on the previous state. Also, the probability of generating each observation depends only on the current state. Using this assumption, the probabilities $P(B \mid K)$ and 
$P(K)$ can be defined as

$$
\begin{aligned}
P(B \mid K) & =\prod_{i=1}^{n} P\left(a_{i} \mid k_{2 i-1}\right) \prod_{i=1}^{n-1} P\left(b_{i} \mid k_{2 i}\right), \\
P(K) & =P\left(k_{1}\right) \prod_{i=2}^{n} P\left(k_{i} \mid k_{i-1}\right) .
\end{aligned}
$$

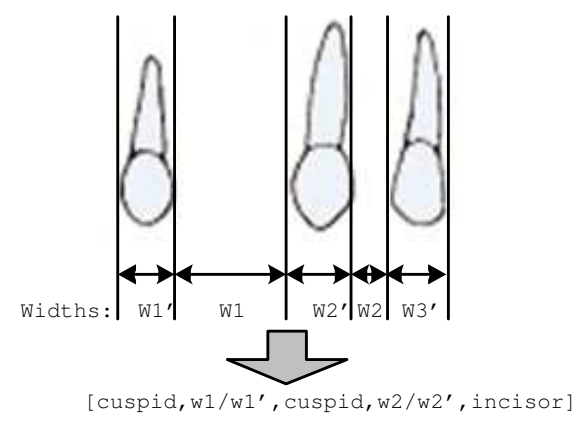

Fig. 5. The generation of an observation sequence.

Note that the value of $a_{i}$ is a discrete number. The probability $P\left(a_{i} \mid k_{m}\right)$ is obtained by using the histogram of $a_{i}$ for state $k_{m}$ computed from the training data. Meanwhile, since the value of $b_{i}$ is a continuous number, we fit a Gaussian model to the training data for the state $k_{m}$ and then $P\left(b_{i} \mid k_{m}\right)$ can be evaluated using the estimated parameters.

Since an observation sequence can start from any tooth state, the probabilities for starting the sequence from any given tooth in the model are the same, i.e., $P(q)=1 / 16$, where $q$ is any one of the 16 tooth states in the model. The optimization of equation (1) uses the Viterbi algorithm [3]. It employs dynamic programming to find the optimum path in the model, which has the largest probability.

\section{TOOTH CLASSIFICATION}

To form the observation sequences, we need to classify the teeth in the radiographs into molars, (bi)cuspids, or incisors. The input to the classifier is the contours of the teeth. Various methods for extraction of the tooth contours have been presented in the literature [4] [5] [1]. Since the radiographs digitized at different times have various scales, the contours extracted from the radiographs need to be normalized. Our aim is to normalize the teeth width so that the average width of non-molars is 100 pixels. Thus, the average width of the non-molars need to be detected beforehand. We fit a mixture of 2 Gaussians to the tooth width histogram. The Gaussian component with the smallest mean value is assumed to represent the width of non-molars, and this value is used as the normalization factor.

We use support vector machines (SVMs) [7] to do classification. SVMs provide better classification accuracy than traditional classifiers in many cases. Since traditional SVMs are 2-class classifiers, for the 3-class classification needed here, we use the 'one-against-one' approach [8]. To improve the classification accuracy, we build three classifiers and their results are combined using the average fusion method [9]. For the fusion, we need to extract the posterior probabilities from the SVMs. This algorithm is presented in $\mathrm{Wu}$ et al.'s work [10].

The input to the first SVM classifier, $C_{1}$, is a vector of $\mathrm{x}$ and $\mathrm{y}$ coordinates of the tooth contours. To obtain the equal length vectors, the raw contours are re-sampled using equal arc-length parameterized splines [11]. The input to the second SVM classifier, $C_{2}$, is the Fourier descriptors of the tooth contours. Fourier descriptors are shape descriptions of curves, which are invariant to translation, scaling and rotation [12]. The input to the third SVM classifier, $C_{3}$, includes several features extracted from tooth contours, including the maximum width of teeth, the minimum width of root end, and the vertical length of tooth contours.

Given a tooth $t$, it is classified as a molar $(m)$, a (bi)cuspid $(c)$, or a incisor $(i)$. The posterior probabilities for the three types of teeth are denoted as $P_{C}(m \mid t), P_{C}(c \mid t)$, and $P_{C}(i \mid t)$, where $C$ is one of the three classifiers, namely, $C_{1}, C_{2}$, or $C_{3}$. By using the average fusion method, the summed likelihood estimates are

$$
\begin{array}{r}
P(m \mid t)=\left(P_{C_{1}}(m \mid t)+P_{C_{2}}(m \mid t)+P_{C_{3}}(m \mid t)\right) / 3, \\
P(c \mid t)=\left(P_{C_{1}}(c \mid t)+P_{C_{2}}(c \mid t)+P_{C_{3}}(c \mid t)\right) / 3, \\
P(i \mid t)=\left(P_{C_{1}}(i \mid t)+P_{C_{2}}(i \mid t)+P_{C_{3}}(i \mid t)\right) / 3 .
\end{array}
$$

The final classification result, $L$, is defined as

$$
L=\arg \max _{L=\{m, c, i\}} P(L \mid t) .
$$

\section{EXPERIMENTAL RESULTS}

To evaluate our algorithm, we have access to a small database from the FBI's Criminal Justice Information Service (CJIS) division, which is interested in utilizing dental characteristics for identifying Missing and Unidentified Persons (MUP). In the database, there are AM and PM dental radiographs belonging to 25 people. A total of 1772 tooth contours were extracted from these radiographs. We must point out that PM dental radiographs are much more difficult to obtain than AM radiographs.

The first experiment dealt with the classification using tooth contours. The radiographs were separated into training data (containing 13 people) and test data (containing 12 people). The implementation of the SVMs used in the experiments was LibSVM (http://www.csie.ntu.edu.tw/ cjlin/libsvm/). Table 1 shows the classification accuracy achieved by each individual classifier and the result of the fusion. Table 2 shows the confusion matrices for the upper and lower teeth after classifier fusion. It is observed 
that upper (bi)cuspids and incisors are more difficult to differentiate than lower (bi)cuspids and incisors. A comparison with the result of Mahoor and Abdel-Mottaleb's method [1] shows that our algorithm, evaluated on a larger database (857 teeth vs. 400 teeth), has a higher classification accuracy $(97.3 \%$ vs. $\sim 90 \%$ for 2 -class problem in [1]).

\begin{tabular}{c|ccc|c}
\hline Classifier & $C_{1}$ & $C_{2}$ & $C_{3}$ & Fusion \\
\hline Upper Teeth & 79.0 & 80.4 & 81.1 & 82.9 \\
Lower Teeth & 90.8 & 89.5 & 93.3 & 93.8 \\
\hline
\end{tabular}

Table 1. Classification accuracies (\%) of individual classifiers and the result of fusion.

\begin{tabular}{c|ccc}
\hline Upper Teeth & Molar & (Bi)cuspid & Incisor \\
\hline Molar & 149 & 13 & 0 \\
(Bi)cuspid & 6 & 167 & 2 \\
Incisor & 8 & 38 & 8 \\
\hline \hline Lower Teeth & Molar & (Bi)cuspid & Incisor \\
\hline Molar & 185 & 6 & 0 \\
(Bi)cuspid & 3 & 197 & 9 \\
Incisor & 0 & 11 & 55 \\
\hline
\end{tabular}

Table 2. Confusion matrices for classification of upper teeth and lower teeth into molars, (bi)cuspids and incisors. The rows are the ground truth of the teeth, and the columns are the results of classifications.

The second experiment dealt with registering the tooth sequences in the radiographs to the dental atlas. There are 562 tooth sequences in the database, belonging to 25 subjects. We randomly chose 8 subjects for training the classifiers, and 8 subjects for training the HMM, and the remaining 9 subjects were used for testing. When training the distance states in the HMM, to make up the deficiency of the sequences that have missing teeth, we artificially generated such sequences from the existing sequences, which do not have missing teeth. To ensure that any tooth that appears in both the radiographs was registered to a single position in the atlas, we concatenated the sequences in the two radiographs into a single sequence as long as the sequences have some teeth in common. This is done by utilizing the information about the common teeth in the radiographs. This resulted in the registration accuracy of $\sim 88 \%$. Figure 6 shows some instances of successful registrations.

The errors in registration were caused by two main reasons: (i) classification errors in short sequences, which had only 3 or 4 teeth; and (ii) erros in detecting the missing teeth. The latter was mainly caused because the distances between neighboring teeth in the testing sequences were not representatives of the training data. These two errors resulted in smaller probabilities of generating the true sequences than generating the imposter sequences, which mis- led the Viterbi algorithm in searching for correct registration positions. Some instances of the registration errors are shown in Fig. 7.

\section{CONCLUSIONS}

A dental atlas is a complete set of indexed adult teeth. This paper presents a method to register a dental radiograph image to the atlas to determine the indices of the teeth in the radiograph. The first stage of the algorithm is to classify the teeth in the radiographs into molars, (bi)cuspids, and incisors. Three SVMs, which use the raw tooth contours, Fourier descriptors of the contours, and other tooth features, respectively, are fused to obtain a good classification accuracy. The second stage uses an HMM to represent the dental atlas. The observed sequences in the radiographs are registered to the atlas by searching for the path that has the largest probability of occurrence. Experimental results show the proposed method is promising. Our future work will utilize the indices of teeth to establish the correspondence of teeth in matching two radiographs for human identification.

\section{REFERENCES}

[1] Mohammad H. Mahoor and Mohamed AbdelMottaleb, "Classification and numbering of teeth in dental bitewing images," to appear in Pattern Recognition.

[2] Paul W. Goaz and Studart C. White, Oral RadiologyPrinciples and Interpretation, The C. V. Mosby Company, St. Louis, 1982.

[3] L. R. Rabiner, "A tutorial on hidden Markov models and selected applications in speech recognition," Proceedings of the IEEE, vol. 77, no. 2, pp. 257-286, 1989.

[4] Anil K. Jain and Hong Chen, "Matching of dental xray images for human identification," Pattern Recognition, vol. 37, no. 7, pp. 1519-1532, 2004.

[5] Hong Chen and Anil K. Jain, "Tooth contour extraction for matching dental radiographs," Proc. 17th ICPR, vol. III, pp. 522-525, Cambridge, UK, August, 2004.

[6] Jindan Zhou and Mohamed Abdel-Mottaleb, "Automatic human identification based on dental x-ray images," in Proc. SPIE Technologies for Homeland Security and Law Enforcement conference, the Biometric Technology for Human Identification conference, Orlando, FL, April 2004.

[7] V. Vapnik, Statistical Learning Theory, Wiley, New York, NY, 1998. 
[8] U. Kressel, "Pairwise classification and support vector machines", in Advances in Kernel Methods - Support Vector Learning, B. Scholkopf, C.J.C. Burges, and A. J. Smola, Eds., pp. 255-268, MIT Press, Cambridge, MA, 1999.

[9] L. I. Kuncheva, "A theoretical study on six classifier fusion strategies," IEEE Trans. Pattern Analysis and Machine Intelligence, vol. 24, no. 2, pp. 281-286, 2002.

[10] T. F. Wu, C. J. Lin, and R. C. Weng, "Probability estimates for multi-class classification by pairwise coupling", in Advances in Neural Information Processing Systems (16), S. Thrun, L. Saul and B. Scholkopf, Eds., MIT Press, Cambridge, MA, 2003.

[11] H. Wang, J. Kearney, and K. Atkinson, "Arc-length parameterized spline curves for real-time simulation," in Proc. 5th International Conference on Curves and Surfaces, San Malo, France, June 2002, pp. 387-396.

[12] P. E. Lestrel, Fourier Descriptors and Their Applications in Biology, Cambridge University Press, USA, 1997.

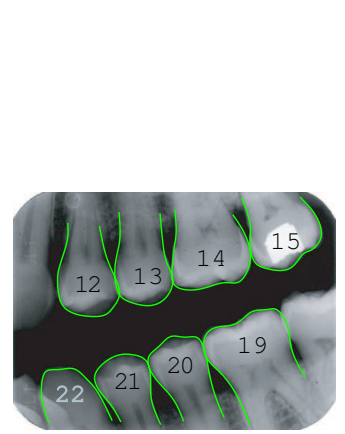

(a)

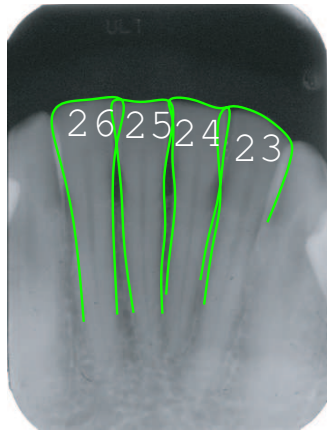

(b)

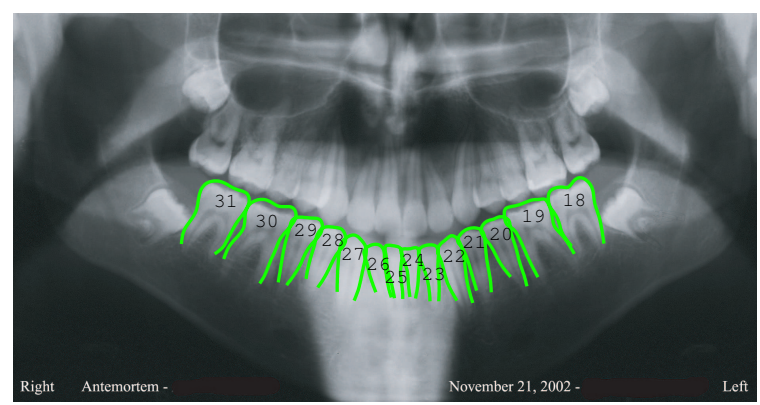

(c)

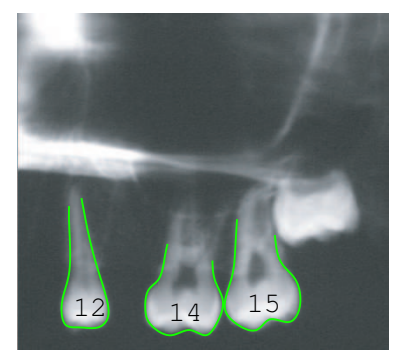

(d)

Fig. 6. Some examples of successful registration of the atlas to (a) a bitewing image, (b) a periapical image, (c) a panoramic image (only the registration to the lower teeth is shown to avoid being huddle), and (d) an image with a missing tooth. In (d), teeth numbered as 12,14 , and 15 are correctly registered. The missing tooth (number 13) is detected. 


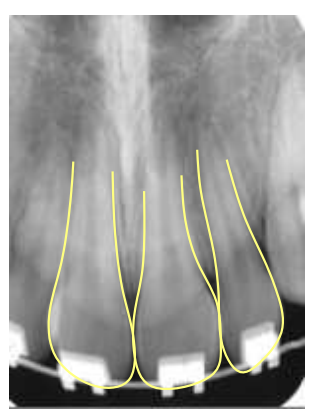

(a)

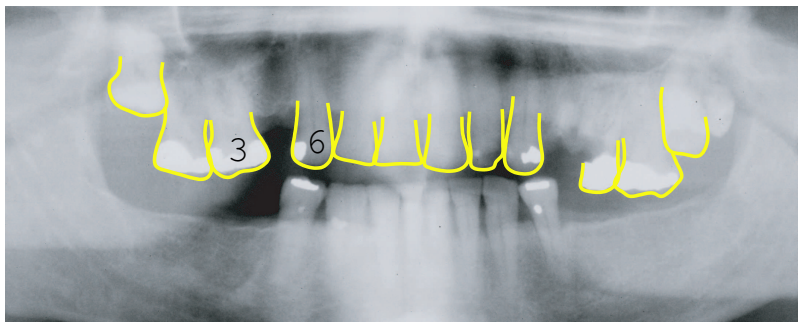

(b)

Fig. 7. Examples of registration errors. (a) All the three incisors are classified as (bi)cuspids, thus these teeth (numbered $8,9,10$ ) are registered to tooth 3,4 and 5 in the atlas. (b) The distance between teeth 3 and 6 supports one missing tooth, so the whole sequence is registered to the atlas tooth numbered $1,2,3,5,6,7,8,9,10,12,13,14$, while the genuine indices are $1,2,3,6,7,8,9,10,11,13,14,15$. The lower row of teeth is correctly registered to the atlas. 